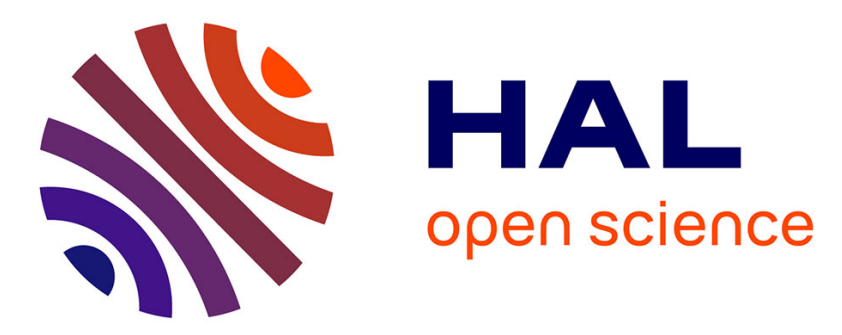

\title{
A quantum mechanical model of electron field emission from two dimensional materials. Application to graphene
}

\author{
Bruno Lepetit
}

\section{- To cite this version:}

Bruno Lepetit. A quantum mechanical model of electron field emission from two dimensional materials. Application to graphene. Journal of Applied Physics, 2021, 129 (14), pp.144302. 10.1063/5.0047771 . hal-03196317

\section{HAL Id: hal-03196317 https://hal.science/hal-03196317}

Submitted on 12 Apr 2021

HAL is a multi-disciplinary open access archive for the deposit and dissemination of scientific research documents, whether they are published or not. The documents may come from teaching and research institutions in France or abroad, or from public or private research centers.
L'archive ouverte pluridisciplinaire HAL, est destinée au dépôt et à la diffusion de documents scientifiques de niveau recherche, publiés ou non, émanant des établissements d'enseignement et de recherche français ou étrangers, des laboratoires publics ou privés. 


\title{
A quantum mechanical model of electron field emission from two dimensional materials. Application to graphene.
}

\author{
Bruno Lepetit* \\ Laboratoire Collisions Agrégats Réactivité, \\ UMR5589, Université de Toulouse, CNRS, \\ Bât. 3R1b4 - 118 route de Narbonne 31062 Toulouse Cedex 09, France
}

(Dated: March 18, 2021)

\begin{abstract}
We implement a new time-independent perturbative quantum method to study quantitatively electron field emission from two dimensional materials and in particular from graphene. The Bardeen transfer Hamiltonian formalism is coupled to a detailed description of the electronic structure of the material. This calculation method is first validated on the standard Fowler-Nordheim (FN) model of a 3 dimensional (3D) free electron gas. Then, it is used to study emission from a 2 dimensional (2D) free electron gas and from graphene represented by a tight-binding model. In the case of graphene, we show that a full electronic band model of the material is necessary to obtain reasonable results because emission is not restricted to the vicinity of the Fermi level near the Dirac points. The graphene emitted current density follows a modified FN law with respect to the applied field, with a prefactor exponent for the field $n \approx 1.5$ intermediate between the one for the cases of 2D $(n=0)$ and 3D $(n=2)$ free electron gases. However, the emitted current level is low because the kinetic energy of the electrons corresponds to a motion parallel to the emitting surface which is not efficient in promoting emission. Our study gives a firm ground to the idea that emission from graphene results almost exclusively from defects.
\end{abstract}




\section{INTRODUCTION}

Electronic field emission from graphene has been the subject of numerous investigations (for reviews, see ref. 1,2). It is commonly assumed that pristine graphene has poor emission properties because its flatness inhibits electric field enhancement. An early experimental evidence of this assumption was given in ref. 3. Emission from different sites of a single layer graphene flake deposited on a $\mathrm{SiO}_{2}$ substrate was measured with a micro-anode probe. It was shown that emission is small near the center of the graphene flake but large near its edges. Therefore, most field emission experiments try to take advantage of morphological characteristics of the structure which enhance locally the applied field and thus the emitted current and many different types of sample preparation which favour the presence of edges, discontinuity and ripples have been implemented ${ }^{2}$. The characteristics of the emitting surface can nowadays be well controlled and correlated to emission properties (for a recent example, see ref. 4).

However, in some experiments, significant current levels were extracted from the central flat part of individual single- and few-layer graphene flakes. For instance, emission currents up to $1 \mu \mathrm{A}$ at applied fields up to $2 \mathrm{~V} / \mathrm{nm}$ were extracted from graphene flakes laid on a flat $\mathrm{SiO}_{2} / \mathrm{Si}$ substrate ${ }^{5}$. Complementary to such studies on supported graphene, others were performed on suspended graphene membranes, which have no direct contact to the substrate except at their rim connected to gold electrodes ${ }^{6}$. It was found that emission is nearly twice larger near its rim than near its center and that the rim turn-on field is only slightly lower than the central one (which is nearly $0.2 \mathrm{~V} / \mathrm{nm}$ ). This means that a significant current can be extracted from all locations of the suspended graphene membrane, although this current remains insufficient to be used in electronic devices ${ }^{7}$. Also, in other recent experiments ${ }^{8}$, field emission with low threshold fields was observed from flat graphene island films deposited on Si wafers in conditions where morphological field enhancement is not expected.

There is thus an apparent disagreement in the litterature on the possibility, or not, to extract significant current from flat graphene. This disagreement is one motivation for the present theoretical study which provides accurate estimates of field emission level from such a material. Another motivation is purely theoretical. The original Fowler-Nordheim model $(\mathrm{FN})^{9}$ and its subsequent refinements ${ }^{10-16}$ still form nowadays the dominant paradigm to understand electronic field emission. These models provide in particular a linear relation 
between $\log \left(I / F^{2}\right)$ and $1 / F$, where $I$ is the emitted current and $F$ the applied electric field. Although widely used successfully over the years, the FN models rely on a crude semi-empirical description of the emitting electrode, represented by a three dimensional (3D) homogeneous electron gas associated to the bulk material, without consideration of its atomic structure. The extension of this type of model to two dimensional (2D) materials like graphene, which consist of one or few atomic layers such that their thickness $L$ is much smaller than their length and width, has been the subject of recent works. A modification of the FN-type model was proposed to describe emission from the edges of such 2D materials ${ }^{3,17}$, it provides a relation between current and field which is different from the usual FN relation. Emissions from the central and edge parts of a suspended graphene membrane were also found to deviate from the usual $\mathrm{FN} \mathrm{law}{ }^{6}$, especially in the low field region. In an attempt to account for these empirical discrepancies with respect to the standard FN law, emission models specifically adapted to flat 2D materials were developed recently ${ }^{18,19}$. The present study reconsiders the problem of the validity of the FN law for 2D materials from an ab initio perspective, taking into account explicitely the atomic structure of the emitting material and its quantum properties.

There is a large set of recent quantum quantitative models ${ }^{20-35}$ (for reviews, see ref. 34,35) available to adress the problem of field emission from materials. We propose here to extend to the present $2 \mathrm{D}$ materials the use of the perturbative methods developped recently ${ }^{36-38}$ to describe emission from metals. We present a time-independent variant of our original timedependent method using the Bardeen transfer Hamiltonian formalism ${ }^{39}$. This formalism has been used on a limited number of problems, like emission from a jellium model of a metal ${ }^{40}$ or carbon nanotubes ${ }^{41}$. In the present paper, we first describe (section II) the formalism which we have implemented and then we describe (section III) the results of its implementation on different materials : first the 3D free electron gas (the usual FN model revisited) to validate the method, then the 2D one and finally the single layer graphene. 


\section{GENERAL METHOD}

\section{A. The Bardeen emission model}

We assume that the cathode surface is in the $\mathbf{x y}$ plane and that it is subjected to an external electric field of amplitude $F(>0)$ parallel to $\mathbf{z}$. The electrons are confined in the material by a potential barrier located near $z_{0}$, to be defined more precisely later. As emitted electrons tunnel only weakly through this barrier, it separates space into 2 domains : the internal domain, where electrons experience the strong electrostatic field induced by the presence of the material nuclei which respect to which the applied electric field screened by the material electrons is negligible ; the external domain, dominated by the external electric field and where the influence of the material electrostatic potential is negligible. At first order, the electron emission is determined by the perturbation operator matrix element coupling unperturbed wavefunctions $\Phi_{\mathbf{k}}(\mathbf{r}, z)(\mathbf{r}=x, y)$ and $\Psi_{\mathbf{k}}(\mathbf{r}, z ; F) . \Phi_{\mathbf{k}}(\mathbf{r}, z)$ is a material valence electron orbital with momentum $\mathbf{k}\left(\mathbf{k}=\mathbf{k}_{\mathbf{x y}}+k_{z} \mathbf{z}\right)$, neglecting the external field. $\Psi_{\mathbf{k}}(\mathbf{r}, z ; F)$ is the continuum state which describes the same electron outside the material, it depends parametrically on the external field $F$ experienced by these emitted electrons. For $2 \mathrm{D}$ materials, $k_{z}=0$ and for $3 \mathrm{D}$ ones, the possible $k_{z}$ values are obtained by applying boundary conditions over the thickness $L$ of the emitting electrode. We consider here for simplicity 0-order diffraction at the interface between the material and vacuum. The component of the momentum parallel to the interface is thus unchanged betwen the material and vacuum orbitals. Notice that the component $k_{z}$ is not conserved in vacuum where electrons are accelerated by the external field. Besides, we neglect all energy exchange between electrons and the lattice, as could occur during collisions between electrons and phonons or impurities. Initial and final states thus have the same energy $\epsilon_{\mathbf{k}}$. As the emitted electrons originate from the valence band, we have : $\epsilon_{0} \leq \epsilon_{\mathbf{k}} \leq \epsilon_{\mathrm{Fermi}}$ where $\epsilon_{0}$ and $\epsilon_{\mathrm{Fermi}}$ are the valence band bottom and top energies.

We assume that the emitting surface consists of $N_{c}$ unit cells, its area is given by : $S=N_{c} A_{p s}$, where $A_{p s}$ is the unit cell area along the material-vacuum interface. The current emitted by $S$ is obtained by summing up the contributions of all the electrons contained in 
$N_{c}$ unit cells and following Bardeen's prescription ${ }^{42}$, it is given by :

$$
I(F)=2 \sum_{\mathbf{k}} I_{\mathbf{k}}(F), \quad I_{\mathbf{k}}(F)=q \frac{2 \pi}{\hbar}\left|\iint_{x, y \in S} d x d y M_{\mathbf{k}}\left(\mathbf{r}, z_{0} ; F\right)\right|^{2}
$$

where the $\mathrm{x} 2$ prefactor accounts for spin degeneracy. The summation on $\mathbf{k}$ is performed over $N_{c}$ points in the first Brillouin zone (1BZ) of the reciprocical space. $q$ is the absolute value of the electron charge and $M_{k}$ is given by :

$$
M_{\mathbf{k}}\left(\mathbf{r}, z_{0} ; F\right)=\frac{\hbar^{2}}{2 m}\left(\Psi_{\mathbf{k}}\left(\mathbf{r}, z_{0} ; F\right)^{*} \frac{d \Phi_{\mathbf{k}}\left(\mathbf{r}, z_{0}\right)}{d z}-\Phi_{\mathbf{k}}\left(\mathbf{r}, z_{0}\right) \frac{d \Psi_{\mathbf{k}}\left(\mathbf{r}, z_{0} ; F\right)^{*}}{d z}\right)
$$

where $\hbar$ is the Planck constant, $m$ is the electron mass and ${ }^{*}$ refers to complex conjugation. We can rewrite the discrete sum in eq. 1 as an integral : $I(F)=2 \times \iint \frac{d^{D} \mathbf{k}}{\delta^{D} \mathbf{k}} I_{\mathbf{k}}(F)$ where $\mathrm{D}=2$ or 3 is the dimensionality of the material. $\delta^{D} \mathbf{k}$ is the elementary area/volume in reciprocical space over which summation is done $: \delta^{D} \mathbf{k}=\frac{(2 \pi)^{D}}{S L^{D-2}}$. The average current density $J(F)$ emitted by $S$ is then :

$$
J(F)=\frac{I(F)}{S}=\frac{L^{D-2}}{2^{D-1} \pi^{D}} \iint d^{D} \mathbf{k} I_{\mathbf{k}}(F)
$$

\section{B. Simplified external wavefunction}

We assume that the external region potential $V_{\text {ext }}(z)$ depends only on $z$. Indeed, the periodic variations of the potential which are large inside the material are quickly damped when the electron moves away from the material into vacuum. The $3 \mathrm{D}$ wavefunction in the external region can be factorized as : $\Psi_{\mathbf{k}}(\mathbf{r}, z ; F)=\frac{1}{S^{\frac{1}{2}}} e^{i \mathbf{k r}} \psi_{\mathbf{k}}(z ; F)$. Inserting this expression and eq. 2 into eq. 1 provides :

$$
I_{\mathbf{k}}(F)=\frac{q}{S} \frac{\pi \hbar^{3}}{2 m^{2}}\left|\psi_{\mathbf{k}}\left(z_{0} ; F\right)\right|^{2}\left|\hat{\Phi}_{\mathbf{k}}\left(z_{0}\right)\right|^{2}\left|\frac{d \hat{\Phi}_{\mathbf{k}}\left(z_{0}\right)}{d z} \hat{\Phi}_{\mathbf{k}}\left(z_{0}\right)^{-1}-\frac{d \psi_{\mathbf{k}}\left(z_{0} ; F\right)}{d z} \psi_{\mathbf{k}}\left(z_{0} ; F\right)^{-1}\right|^{2}
$$

$\hat{\Phi}_{\mathbf{k}}$ being the 2D Fourier transform of $\Phi_{\mathbf{k}}(\mathbf{r}, z)$ :

$$
\hat{\Phi}_{\mathbf{k}}(z)=\iint d x d y e^{-i \mathbf{k r}} \Phi_{\mathbf{k}}(\mathbf{r}, z) .
$$

We now assume that the external potential $V_{\text {ext }}(z)$ is linear : $V_{\text {ext }}(z)=V_{\infty}-q F\left(z-z_{i}\right)$. Solving the one dimensional Poisson equation for a uniform conducting plane subjected to an external static field shows that $z_{i}$ is the induced charge centroid ${ }^{43}$. It is also the image plane location for an external test electron ${ }^{44}$, but not for one of the substrate which experiences 
the additional exchange interaction with the other substrate electrons ${ }^{45}$. The solution of the one dimensional Schrödinger equation in the external domain :

$$
\left(-\frac{\hbar^{2}}{2 m} \frac{d^{2}}{d z^{2}}+V_{e x t}(z)\right) \psi_{\mathbf{k}}(z ; F)=\left(\epsilon_{\mathbf{k}}-\frac{\hbar^{2} \mathbf{k}_{\mathbf{x y}}{ }^{2}}{2 m}\right) \psi_{\mathbf{k}}(z ; F)
$$

is known analytically :

$$
\psi_{\mathbf{k}}(z ; F)=N A i\left(-\left(\frac{2 m q F}{\hbar^{2}}\right)^{\frac{1}{3}}\left(z-z_{T}(\mathbf{k}, F)\right)\right)
$$

$A i$ is an Airy function ( $\$ 10.4$ in ref. 46$), N$ a normalization constant, $z_{T}(\mathbf{k}, F)$ is the turning point defined implicitly by : $V_{e x t}\left(z_{t}(\mathbf{k}, F)\right)=\epsilon_{\mathbf{k}}-\frac{\hbar^{2} \mathbf{k}_{\mathbf{x y}}{ }^{2}}{2 m}$ and explicitly by :

$$
z_{T}(\mathbf{k}, F)=z_{i}+\frac{W_{\mathbf{k}}}{q F}
$$

with :

$$
W_{\mathbf{k}}=V_{\infty}-\left(\epsilon_{\mathbf{k}}-\frac{\hbar^{2} \mathbf{k}_{\mathbf{x y}}{ }^{2}}{2 m}\right)=W+W_{B}+\frac{\hbar^{2} \mathbf{k}_{\mathbf{x y}}{ }^{2}}{2 m}-\Delta \epsilon_{\mathbf{k}} ; \Delta \epsilon_{\mathbf{k}}=\epsilon_{\mathbf{k}}-\epsilon_{\mathbf{0}}
$$

$W_{\mathbf{k}}$ is the energy which must be given to an electron initially in the energy level $\epsilon_{\mathbf{k}}$ to escape into the vacuum level $V_{\infty}+\frac{\hbar^{2} \mathbf{k}_{\mathbf{x y}}}{2 m}$, with sufficient energy to sustain its motion parallel to the interface with momentum $\mathbf{k}_{\mathbf{x y}} . W=V_{\infty}-\epsilon_{\text {Fermi }}$ is the work function, $W_{B}=\epsilon_{\text {Fermi }}-\epsilon_{\mathbf{0}}$ the band width. $\epsilon_{\text {Fermi }}$ and $\epsilon_{\mathbf{0}}$ are the valence band top (Fermi level) and bottom energies. The continuum function $\psi_{\mathbf{k}}(z)$ is normalized by the prescription : $\int_{-\infty}^{+\infty} d z \psi_{\mathbf{k}^{\prime}}(z ; F)^{*} \psi_{\mathbf{k}}(z ; F)=$ $\delta\left(\epsilon_{\mathbf{k}}-\epsilon_{\mathbf{k}^{\prime}}\right)$ where $\delta$ refers to the Dirac distribution. Applying the method described in appendix A of ref. 47 and using the asymptotic forms of $A i$ given for the vacuum side by eq. 10.4.78 and 10.4.79 of ref. 46, we obtain : $N=\frac{(2 m)^{\frac{1}{3}}}{\hbar^{\frac{2}{3}}(q F)^{\frac{1}{6}}}$. The barrier near $z_{0}$ is located in the energetically forbidden region of the potential where we can replace with an excellent approximation the Airy function by its asymptotic form given for the material side by eq. 10.4.59 of ref. 46 so that $\psi_{\mathbf{k}}\left(z_{0} ; F\right)$ can be written as :

$$
\psi_{\mathbf{k}}\left(z_{0} ; F\right) \approx \frac{(2 m)^{\frac{1}{4}}}{2 \hbar^{\frac{1}{2}} \pi^{\frac{1}{2}}(q F)^{\frac{1}{4}}\left(z_{T}(\mathbf{k}, F)-z_{0}\right)^{\frac{1}{4}}} e^{-\frac{2}{3}\left(\frac{2 m q F}{\hbar^{2}}\right)^{\frac{1}{2}}\left(z_{T}(\mathbf{k}, F)-z_{0}\right)^{\frac{3}{2}}}
$$

A similar expression can be obtained for $\frac{d \psi_{\mathbf{k}}\left(z_{0} ; F\right)}{d z}$ using the asymptotic form given by eq. 10.4.61 in ref. 46. Inserting these expressions in eq. 4 and choosing the observation plane as the induced charge centroid plane : $z_{0}=z_{i}$, we finally obtain :

$$
I_{\mathbf{k}}(F)=\frac{q}{S} \frac{\hbar^{2}\left|\hat{\Phi}_{\mathbf{k}}\left(z_{i}\right)\right|^{2}}{2^{\frac{5}{2}} m^{\frac{3}{2}} W_{\mathbf{k}}^{\frac{1}{2}}}\left|\frac{d \hat{\Phi}_{\mathbf{k}}\left(z_{i}\right)}{d z} \hat{\Phi}_{\mathbf{k}}\left(z_{i}\right)^{-1}-\left(\frac{2 m W_{\mathbf{k}}}{\hbar^{2}}\right)^{\frac{1}{2}}\right|^{2} e^{-\frac{4}{3}\left(\frac{2 m}{\hbar^{2}}\right)^{\frac{1}{2}} \frac{W_{\mathbf{k}}^{\frac{3}{2}}}{q F}}
$$


We now show how to apply this general relation in conjunction with eq. 3 to study emission properties of different materials.

\section{APPLICATION TO DIFFERENT MATERIAL TYPES}

\section{A. The 3 dimensional free electron gas : the Fowler-Nordheim model revisited}

In order to validate the present formalism, we first apply it to the material of the FowlerNordheim model. It consists of a 3D free electron gas with energy : $\epsilon_{\mathbf{k}}=\frac{\hbar^{2} \mathbf{k}_{\mathbf{x y}}{ }^{2}}{2 m}+\frac{\hbar^{2} k_{z}^{2}}{2 m}+$ $\epsilon_{0}$ so that (eq. 9): $W_{\mathbf{k}}=W+W_{B}-\frac{\hbar^{2} k_{z}^{2}}{2 m}$. Since the electron gas is uniform in the material, we have : $\left|\hat{\Phi}_{\mathbf{k}}\left(z_{i}\right)\right|^{2}=\frac{S}{L}$ and as its wavefunction decays exponentially in vacuum : $\frac{d \hat{\Phi}_{\mathbf{k}}\left(z_{i}\right)}{d z} \hat{\Phi}_{\mathbf{k}}\left(z_{i}\right)^{-1}=-\left(\frac{2 m}{\hbar^{2}}\right)^{\frac{1}{2}} W_{\mathbf{k}}^{\frac{1}{2}}$. As a result, $I_{\mathbf{k}}(F)$ depends on $k_{z}$ only, not on $\mathbf{k}_{\mathbf{x y}}$ (eq. 11$)$. Integrating over $k_{x}, k_{y}$ in eq. 3 is therefore straightforward and amounts in evaluating the area of the disk inside the sphere $\Delta \epsilon_{\mathbf{k}} \leq W_{B}$ in the $\left(k_{x}, k_{y}\right)$ plane corresponding to a given $k_{z}$. The result is :

$$
J(F)=\frac{q m^{\frac{1}{2}}}{2^{\frac{1}{2}} \pi^{2} \hbar^{2}} \int_{0}^{\left(\frac{2 m W_{B}}{\hbar^{2}}\right)^{\frac{1}{2}}} d k_{z} W_{\mathbf{k}}^{\frac{1}{2}}\left(W_{\mathbf{k}}-W\right) e^{-\frac{4}{3}\left(\frac{2 m}{\hbar^{2}}\right)^{\frac{1}{2}} \frac{W_{\mathbf{k}}^{\frac{3}{2}}}{q F}}
$$

Calculating the integral involves, as in the standard Fowler-Nordheim model, a first order Taylor expansion of the integrand in the vicinity of the Fermi level. The final result is :

$$
J(F)=\frac{q^{3} F^{2}}{16 \pi^{2} \hbar\left(W W_{B}\right)^{\frac{1}{2}}} e^{-\frac{4}{3}\left(\frac{2 m}{\hbar^{2}}\right)^{\frac{1}{2}} \frac{W^{\frac{3}{2}}}{q F}}
$$

This expression is identical to the standard Fowler-Nordheim result (with $n=2$ ):

$$
J(F)=\frac{q^{3} F^{n}}{16 \pi^{2} \hbar W} e^{-\frac{4}{3}\left(\frac{2 m}{\hbar^{2}}\right)^{\frac{1}{2}} \frac{W^{\frac{3}{2}}}{q F}}
$$

if we replace $W$ by $\left(W W_{B}\right)^{\frac{1}{2}}$ in the prefactor. As both terms have the same order of magnitude, it turns out that both models provide very similar results. In particular, the usual linear dependence of $\log \left(J(F) / F^{2}\right)$ with respect to $1 / F$ is recovered. The similarity between both results is remarkable because they have been obtained with two completely different formalisms. The Bardeen formalism indeed uses a perturbative quantum method whereas the Fowler Nordheim model relies on a semi-classical non-perturbative one. Our result thus validates the use of the Bardeen method for electronic emission problems and allows to use it for problems more complex than permits the implementation of the FowlerNordheim model. 


\section{B. The 2 dimensional free electron gas}

The material wavefunction now reads : $\Phi_{\mathbf{k}}(\mathbf{r}, z)=\frac{1}{S^{\frac{1}{2}}} e^{i \mathbf{k}_{\mathbf{x y}} \mathbf{r}} \varphi(z)$, where $\varphi(z)$ is a bound state with energy $\epsilon_{0}$. We have $: \epsilon_{\mathbf{k}}=\frac{\hbar^{2} \mathbf{k}_{\mathbf{x y}}{ }^{2}}{2 m}+\epsilon_{0}$ and (eq. 9) $: W_{\mathbf{k}}=W+W_{B} . I_{\mathbf{k}}(F)$ is thus independent of $\mathbf{k}$ (eq. 11) and integration (eq. 3) is straighforward :

$$
J(F)=\frac{q(2 m)^{\frac{1}{2}}}{\pi \hbar^{2}} W_{B}\left(W+W_{B}\right)^{\frac{1}{2}}\left|\varphi\left(z_{i}\right)\right|^{2} e^{-\frac{4}{3}\left(\frac{2 m}{\hbar^{2}}\right)^{\frac{1}{2}} \frac{\left(W+W_{B}\right)^{\frac{3}{2}}}{q F}}
$$

In going from the 3D to the 2D free electron gas, the $F^{2}$ pre-factor is lost as the field exponent changes from $n=2$ to $n=0 . \log J(F)$ is linearly dependent with respect to $1 / F$, with a slope controlled by $W+W_{B}$ in the $2 \mathrm{D}$ case, the energy necessary to extract an electron from the band bottom, instead of by $W$, the work function necessary to extract an electron from the band top, in the 3D case. Emission from 2D materials is thus disfavored as compared to $3 \mathrm{D}$ ones. This is of course related to the fact that the kinetic energy associated to the electron motion parallel to the interface is not efficient in promoting emission.

\section{Graphene}

\section{Tight-binding model}

We now look for a simple expression for the wavefunction $\hat{\Phi}_{\mathbf{k}}\left(z_{i}\right)$ to insert in eq. 11 in the case of graphene. This wavefunction depends on the atomic structure of the material. Graphene is a honeycomb crystal lattice which contains two atoms per unit cell. Each belongs to a different sub-lattice, A or B, each A type atom being surrounded by three $\mathrm{B}$ type ones, and vice-versa (see fig. 1). The lattice vectors are given by : $\mathbf{a}_{\mathbf{1}}=\frac{a}{2}(3, \sqrt{3}), \quad \mathbf{a}_{\mathbf{2}}=\frac{a}{2}(3,-\sqrt{3})$ where $a$ is the nearest-neighbor distance. The vectors connecting one atom (of one type) to its neighbors (of the other type) are given by : $\delta_{\mathbf{1}}=\frac{a}{2}(1, \sqrt{3}), \quad \delta_{\mathbf{2}}=\frac{a}{2}(1,-\sqrt{3}), \quad \delta_{\mathbf{3}}=a(-1,0)$. The graphene unit cells are labelled by the position vectors $\mathbf{r}_{\mathbf{L}}$ given by : $\mathbf{r}_{\mathbf{L}}=n_{1} \mathbf{a}_{\mathbf{1}}+n_{2} \mathbf{a}_{\mathbf{2}}$, where $n_{1}$ and $n_{2}$ are integers. The positions of the atoms in each cell are given by $\mathbf{r}_{\mathbf{L}}{ }^{s}=\mathbf{r}_{\mathbf{L}}+\delta^{s}(s=A$ or $B)$ with $\delta^{A}=(0,0)$ and $\delta^{B}=(a, 0)$ ( $s$ being the sublattice index $s=A$ or $B$ ). The unit cell area in physical space is given by : $A_{p s}=\frac{3 \sqrt{3}}{2} a^{2}$.

We use the tight-binding formalism which combines simplicity with accuracy ${ }^{48,49}$. A 
graphene orbital $\Phi_{\mathbf{k}}$ is given by :

$$
\Phi_{\mathbf{k}}(\mathbf{r}, z)=a_{\mathbf{k}} \Phi_{\mathbf{k}}^{A}(\mathbf{r}, z)+b_{\mathbf{k}} \Phi_{\mathbf{k}}^{B}(\mathbf{r}, z)
$$

The functions $\Phi_{\mathbf{k}}^{s}(\mathbf{r}, z)$ associated to each sublattice $A$ or $B$ can be expanded in Wannier states localized in the vicinity of the atoms positions $\mathbf{r}_{\mathbf{L}}+\delta^{s}$ :

$$
\Phi_{\mathbf{k}}^{s}(\mathbf{r}, z)=\frac{1}{\sqrt{N_{c}}} \sum_{\mathbf{r}_{\mathbf{L}}} e^{i \mathbf{k r}_{\mathbf{L}}} \phi\left(\mathbf{r}-\left(\mathbf{r}_{\mathbf{L}}+\delta^{s}\right), z\right)
$$

This corresponds to a Fourier series expansion of a function which is periodic in reciprocical space. The tight-binding approximation consists in assuming that $\phi$ is a carbon pz atomic orbital, to be described in more details later. Inserting eq. 16 and 17 in eq. 11, and introducing $\hat{\phi}_{\mathbf{k}}(z)$, the 2D Fourier transform (eq. 5) of $\phi(\mathbf{r}, z)$, we obtain :

$$
\begin{aligned}
& I_{\mathbf{k}}(F)=\frac{q}{A_{p s}} \times \\
& \frac{\hbar^{2}\left|a_{\mathbf{k}} e^{-i \mathbf{k} \delta^{A}}+b_{\mathbf{k}} e^{-i \mathbf{k} \delta^{B}}\right|^{2}\left|\hat{\phi}_{\mathbf{k}}\left(z_{i}\right)\right|^{2}}{2^{\frac{5}{2}} m^{\frac{3}{2}} W_{\mathbf{k}}^{\frac{1}{2}}}\left|\frac{d \hat{\phi}_{\mathbf{k}}\left(z_{i}\right)}{d z} \hat{\phi}_{\mathbf{k}}\left(z_{i}\right)^{-1}-\left(\frac{2 m}{\hbar^{2}}\right)^{\frac{1}{2}} W_{\mathbf{k}}^{\frac{1}{2}}\right|^{2} e^{-\frac{4}{3}\left(\frac{2 m}{\hbar^{2}}\right)^{\frac{1}{2}} \frac{W_{\mathbf{k}}^{\frac{3}{2}}}{q F}}
\end{aligned}
$$

The pair of coefficients $a_{\mathbf{k}}, b_{\mathbf{k}}$ is an eigenvector of the $2 \times 2$ tight-binding Hamiltonian $\mathbf{H}(\mathbf{k})$. In its simplest form, overlaps are assumed to be 0 between orbitals centered on different atoms and couplings are assumed to be non-zero only between nearest neighbors :

$$
\mathbf{H}(\mathbf{k})=\left(\begin{array}{cc}
e & t \gamma(\mathbf{k})^{*} \\
t \gamma(\mathbf{k}) & e
\end{array}\right)
$$

where $t(>0)$ is the hopping parameter, $e$ the carbon atom orbital energy and : $\gamma(\mathbf{k})=$ $1+e^{i \mathbf{k a}_{1}}+e^{i \mathbf{k} \mathbf{a}_{2}}$. The energy of the graphene orbital $\Phi_{\mathbf{k}}(\mathbf{r}, z)$ belonging to the valence band is the lowest eigenvalue of this Hamiltonian and is given by : $\epsilon_{\mathbf{k}}=e-t|\gamma(\mathbf{k})|$ with $:|\gamma(\mathbf{k})|=$ $\sqrt{3+2\left(\cos \left(\sqrt{3} k_{y} a\right)+2 \cos \left(k_{x} \frac{3 a}{2}\right) \cos \left(k_{y} \frac{\sqrt{3} a}{2}\right)\right)}$ The $\Gamma$ point $\left(\mathbf{k}_{\Gamma}=(0,0)\right)$ corresponds to the electronic band bottom : $\epsilon_{\mathbf{k}_{\Gamma}}=e-3 t$; the M point $\left(\mathbf{k}_{M}=\left(\frac{2 \pi}{3 a}, 0\right)\right)$ to $: \epsilon_{\mathbf{k}_{M}}=e-t$, the $\mathrm{K}$ point $\left(\mathbf{k}_{K}=\left(\frac{2 \pi}{3 a}, \frac{2 \pi}{3 \sqrt{3} a}\right)\right)$, the apex of the Dirac cone, to $: \epsilon_{\mathbf{k}_{K}}=e=\epsilon_{\text {Fermi }}$. The atomic orbital energy is also the Fermi energy.

The eigenvector $a_{\mathbf{k}}, b_{\mathbf{k}}$ is given by : $a_{\mathbf{k}}=-\frac{1}{\sqrt{2}} \frac{\gamma(\mathbf{k})^{*}}{\gamma(\mathbf{k}) \mid}, b_{\mathbf{k}}=\frac{1}{\sqrt{2}}$. Eq. 18 can thus be rewritten :

$$
I_{\mathbf{k}}(F)=C_{\mathbf{k}} e^{-\frac{4}{3}\left(\frac{2 m}{\hbar^{2}}\right)^{\frac{1}{2}} \frac{W_{k}^{\frac{3}{2}}}{q F}}
$$


with $C_{\mathbf{k}}$ a $F$-independent coefficient :

$$
C_{\mathbf{k}}=\frac{q}{A_{p s}} \frac{\hbar^{2}\left|\hat{\phi}_{\mathbf{k}}\left(z_{i}\right)\right|^{2}}{2^{\frac{5}{2}} m^{\frac{3}{2}} W_{\mathbf{k}}^{\frac{1}{2}}}\left(1-\frac{\beta(\mathbf{k})}{|\gamma(\mathbf{k})|}\right)\left|\frac{d \hat{\phi}_{\mathbf{k}}\left(z_{i}\right)}{d z} \hat{\phi}_{\mathbf{k}}\left(z_{i}\right)^{-1}-\left(\frac{2 m}{\hbar^{2}}\right)^{\frac{1}{2}} W_{\mathbf{k}}^{\frac{1}{2}}\right|^{2}
$$

with : $\beta(\mathbf{k})=\cos \left(k_{x} a\right)+2 \cos \left(k_{x} \frac{a}{2}\right) \cos \left(k_{y} \frac{\sqrt{3} a}{2}\right)$. Inserting eq. 21 in eq. 20 , and then eq. 20 into eq. 3 , we can compute the emitted current density.

\section{Numerical application}

We use the tight-binding data given in ref. 48 : the carbon atom nearest neighbor distance is $a=0.142 \mathrm{~nm}$ and the nearest neighbor hopping energy is $t=2.8 \mathrm{eV}$ so that the band width is : $W_{B}=3 t=8.4 \mathrm{eV}$. The resulting energy spectrum $\epsilon_{\mathbf{k}}$ is shown on fig. 2 (top) for the valence band in the $k_{x}, k_{y}>0$ portion of the 1BZ. The $\mathrm{K}$ and $\mathrm{K}$ ' points are 2 of the 6 equivalent locations of the double inverted cone where the valence band touches the conduction one, the so called Dirac points, as expected for a semi-metal. In the vicinity of the $\Gamma$ point, the spectrum has the expected parabolic shape given by $: \Delta \epsilon_{\mathbf{k}} \approx \frac{\hbar^{2} \mathbf{k}_{\mathbf{x y}}{ }^{2}}{2 m_{\Gamma}}$. The effective mass is obtained by a simple second order Taylor expansion of $\Delta \epsilon_{\mathbf{k}}: m_{\Gamma}=\frac{2 \hbar^{2}}{3 t a^{2}} \approx$ $0.9 \mathrm{~m}$. Graphene electrons have lower mass than free ones because they are more mobile by hopping between atomic sites. However, further away from the $\Gamma$ point, $\Delta \epsilon_{\mathbf{k}}$ becomes increasingly smaller than $\frac{\mathbf{k}_{\mathbf{x y}}{ }^{2}}{2 m_{\Gamma}}$ and even smaller than $\frac{\mathbf{k}_{\mathbf{x y}}{ }^{2}}{2 m}$. This impacts the shape of the $W_{\mathbf{k}}$ function (eq. 9) shown in the $1 \mathrm{BZ}$ on fig. 2 (bottom) for : $W=4.5 \mathrm{eV}$ following ref. 50 . In the vicinity of the $\Gamma$ point, we have : $W_{\mathbf{k}} \approx\left(W+W_{B}\right)+\frac{\hbar^{2} \mathbf{k}_{\mathbf{x y}}^{2}}{2}\left(\frac{1}{m}-\frac{1}{m_{\Gamma}}\right)<W+W_{B}$. The $\Gamma$ point corresponds to a relative maximum of $W_{\mathbf{k}}$, the absolute one being on the M-K-K' line. The minimum region separates $\Gamma$ from M-K-K'. As electronic emission is controlled by $W_{\mathbf{k}}$ mainly through the exponential factor (eq. 20), highest emission is expected from electrons with momentum $\mathbf{k}$ from the region where $W_{\mathbf{k}}$ is minimum. Thus, counter intuitively, the vicinity of the $\mathrm{K}$ point, which corresponds to the Fermi level, is not expected to contribute significantly to emission. Instead, significant emission is expected in the vicinity of the annular region around the $\Gamma$ point : $|\mathbf{k}| \approx 6 \mathrm{~nm}^{-1}$, which is the region of $W_{\mathbf{k}}$ minima: $W_{\mathbf{k}}^{\min }=12.81 \mathrm{eV}$.

Fig. 3 shows the current distribution $I_{\mathbf{k}}(F)$ (eq. 20) in the 1BZ, the image plane location being : $z_{i}=0.1 \mathrm{~nm}$, following ref. 51-53. A simple Slater type representation of the 
carbon $p z$ orbitals is used (ref. 54, p. 802 and p. 1145) $: \phi(\mathbf{r}, z)=\frac{(6-s)^{\frac{5}{2}} z}{\left(2 a_{0}\right)^{\frac{5}{2}} \pi^{\frac{1}{2}}} e^{-\frac{(6-s) \rho}{2 a_{0}}}$ with $a_{0}$, the Bohr radius, defined by : $a_{0}=\frac{4 \pi \epsilon_{0} \hbar^{2}}{m q^{2}}$ and $\rho=\left(\mathbf{r}^{2}+z^{2}\right)^{\frac{1}{2}}$. Optimized screening constants following ref. 55 provide $: s=2.8642$. The orbital 2D Fourier transform (eq. 5 ) is performed numerically. Maximum emission is observed from an annular region surrounding the $\Gamma$ point, at a distance $|\mathbf{k}| \approx 9 \mathrm{~nm}^{-1}$ larger than expected $\left(|\mathbf{k}| \approx 6 \mathrm{~nm}^{-1}\right)$. Indeed, the dependence of $I_{\mathbf{k}}(F)$ on $\mathbf{k}$ results not only from the exponential factor but also from the prefactor $C_{\mathbf{k}}$ which is an increasing function of $|\mathbf{k}|$ (eq. 21). Also, mainly because of the $1-\frac{\beta(\mathbf{k})}{|\gamma(\mathbf{k})|}$ factor related to the geometry of the lattice, $C_{\mathbf{k}}$ and $I_{\mathbf{k}}(F)$ have a strongly directional character in reciprocical space. As a result, emission is modulated with respect to direction in the emitting annular region and is localized near the 6 points $\mathbf{k}_{\mathbf{0}}^{\mathbf{i}}(\mathbf{i}=1-6)$ obtained from $\mathbf{k}_{0}^{1}=\left(k_{0 x}^{1} \approx 9 \mathrm{~nm}^{-1}, k_{0 y}^{1}=0\right)$ by rotations of angle $\frac{(i-1) \pi}{3}$ around $\Gamma$. We thus obtain for field emission a result already observed for thermoionic emission ${ }^{56}$, namely that emission involves electrons residing far away from the Dirac K points. This justifies a posteriori the present use of a graphene full band model, instead of a linear approximation which would be valid in the vicinity of the Dirac points only.

Integration of $I_{\mathbf{k}}(F)$ performed by numerical quadrature over a $100 \times 100$ grid in the $k_{x}, k_{y}>0$ portion of the $1 \mathrm{BZ}$ provides the emitted current density as a function of the applied electric field (eq. 3), as shown on fig. 4. The obtained currents are very small. Similarly to the 2D free electron gas problem (section IIIB), the energy carried by the graphene electrons corresponds mainly to a motion parallel to the interface and it is not efficient in promoting their emission.

\section{Analytical approximation}

Recent studies considered the functional relation between current and applied field in the case of low dimensionality materials ${ }^{18,19}$. The applicability for such materials of the Fowler-Nordheim law (eq. 14) with the usual exponent $n=2$ is one the main issues raised in these studies. In the case of the $2 \mathrm{D}$ free electron gas, we found $n=0$ (see section III B) and we had to substitute $W+W_{B}$, the vacuum level energy with respect to the band bottom energy, to the usual work function $W$ in the exponential argument. In order to answer this question in the case of graphene, we look for an analytical approximation of the emitted current computed numerically using eq. 3 (section III C 2). We have shown 
that emission is localized near the 6 equivalent points $\mathbf{k}_{\mathbf{0}}^{\mathbf{i}}(\mathbf{i}=1-6)$ in the 1BZ. Assuming for simplicity that $C_{\mathbf{k}}$ is constant with respect to $\mathbf{k}$, then these points coincide with the minima of $W_{\mathbf{k}}$. Performing a second order Taylor expansion of $I_{\mathbf{k}}(F)$ in the vicinity of these points in eq. 20, eq. 3 becomes : $J(F) \approx \frac{9}{4 \pi}\left(\frac{\hbar^{2}}{2 m}\right)^{\frac{1}{2}} \frac{C_{\mathbf{k}_{0}^{1}}}{\left(\left(W_{\mathbf{k}_{0}^{1}}^{\frac{3}{2}} x^{x x}\left(W_{\mathbf{k}_{0}^{2}}^{\frac{3}{2}}\right)^{y y}\right)^{\frac{1}{2}}\right.} q F e^{-\frac{4}{3}\left(\frac{2 m}{\hbar^{2}}\right)^{\frac{1}{2}} \frac{W_{\mathbf{k}_{0}^{1}}^{\frac{3}{2}}}{q F}}$ using the second derivatives: $\left(W_{\mathbf{k}_{\mathbf{0}}^{1}}^{\frac{3}{2}}\right)^{x x}=\frac{d^{2}}{d k_{x}^{2}}\left(W_{\mathbf{k}_{\mathbf{0}}^{1}}^{\frac{3}{2}}\right),\left(W_{\mathbf{k}_{\mathbf{0}}^{1}}^{\frac{3}{2}}\right)^{y y}=\frac{d^{2}}{d k_{y}^{2}}\left(W_{\mathbf{k}_{\mathbf{0}}^{1}}^{\frac{3}{2}}\right)$. This suggests a linear dependence between $\log (J(F) / F)$ and $1 / F$, the slope of which is controlled by $W_{\mathbf{k}_{0}^{1}}$. Since $W \ll W_{\mathbf{k}_{\mathbf{0}}^{1}} \lesssim W+W_{B}$, emission from graphene is expected to be smaller than from graphite, but slightly larger than from a $2 \mathrm{D}$ free electron gas (eq. 15), if we consider the exponential factor only.

This result is based on the crude assumption that $C_{\mathbf{k}}$ is constant. It provides however a useful guideline for a fit. The best fit of the numerical emitted current density $J(F)$ using eq. 14 with fixed $n=1$ and with $W$ as the only free fitting parameter was obtained for $W=12.86 \mathrm{eV}$, which is close to the minimum of $W_{\mathbf{k}}$ in reciprocical space : $W_{\mathbf{k}}^{\min }=12.81 \mathrm{eV}$ (see section III C 2). The relative error between the numerical and the fitted results is better than $20 \%$ (see fig. 4). We also performed a fit with fixed $n=2$ (the usual Fowler-Nordheim prefactor), we obtained $W=12.71 \mathrm{eV}$ and a relative error also better than $20 \%$. Now allowing both the exponent $n$ and $W$ to be free fitting parameters, we obtained the best fit for $n=1.53$ and $W=12.78 \mathrm{eV}$. The relative error is then, as expected, much lower ( $1 \%$ or better). A power law with $n \approx 1.5$, intermediate between the $2 \mathrm{D}$ and $3 \mathrm{D}$ free electron gas cases, is thus found to provide the most representative pre-factor for graphene. The same $n=\frac{3}{2}$ power dependence was obtained theoretically for the emission from a graphene nanowall in the high field limit ${ }^{17}$. A nanowall corresponds to a graphene flake mounted perpendicularly to the substrate material and in this case, emitted electrons escape from graphene parallel to the flake surface through its rim. The same $n=\frac{3}{2}$ power dependence was also obtained in the experimental study of ref. 3 from a fit of emitted current measurement at high fields ( $n=3$ was obtained at low fields which are not considered in the present study). The fact that the same power dependence is obtained for both emission configurations, parallel (ref. 17) or perpendicular (present work) to the graphene sheet, prevents from deducing from the measured power dependence what was the graphene emission configuration in the experiment. Very recently ${ }^{57}$, a different $n=1$ power dependence was obtained for the general class of 2D semi-metals to which graphene belongs. A possible reason, among others, for 
this discrepancy may be related to differences in the graphene dispersion relation : whereas the present one is global and valid in the whole reciprocical space, the one used in ref. 57 is a local one, valid in the vicinity of the Dirac K point only.

\section{CONCLUSION}

We have presented in this paper an implementation of the general Bardeen formalism to study 2D material field emission. This formalism uses a quantum mechanical perturbative method and is based on the detailed knowledge of the material at the atomi level. We have considered the 2D free electron gas and graphene problem. Modified Fowler-Nordheim laws were shown to be good approximations of the emitted current dependence with respect to the applied field. The modifications concern the field exponent in the pre-factor : $n=0$ for the $2 \mathrm{D}$ free-electron gas and $\mathrm{n} \approx 1.5$ for graphene, as compared to $n=2$ for the standard Fowler-Nordheim law. Also, the exponential argument depends on the difference of energy between the vacuum and the valence band bottom energies in the case od 2D materials, instead of the band top (Fermi level) for 3D ones. In the case of graphene, emission was shown to involve mainly electrons with low momentum parallel to the interface, instead of electrons close in energy to the Fermi level (Dirac points) which have high momentum. But from a general point a view, 2D materials are not good emitters simply because the electron motion parallel to the interface in the material does not favor it. The present model corresponds, strictly speaking, to suspended graphene, not taking into account edge emission. However, as supported graphene interacts weakly with its substrate, these results are expected to be valid in this case also. It is thus clear that the significant emitted levels observed experimentally originate from defects (ripples, contaminations, edges...) in the planarity of the materials.

Although our results are $a b$ initio ones and do not contain empirical adjustments, they involve physical approximations which it may be interesting to relax. For instance, the tight-binding graphene orbitals which we used do not have a carefully designed asymptotic behavior far from the surface. Higher quality orbitals could be used. More fundamentally, we assumed conservation of the electron momentum parallel to the interface, neglecting diffraction and dissipation induced by electron-phonon interactions. However, although such improvements would modify the quantitative results presented here, we do not believe 
that they would change the physical conclusions which were reached. Notice finally that the present results may be useful not just to study field emission, but also charge injection in 2D material $\operatorname{contacts}^{58}$.

\section{DATA STATEMENT}

The data that support the findings of this study are available from the corresponding author upon reasonable request. 
* Electronic address: bruno.lepetit@irsamc.ups-tlse.fr

1 L. Chen, H. Yua, J. Zhong, L. Song, J. Wuc, and W. Su, Materials Science and Engineering B, 220, 44 (2017).

2 F. Giubileo, A. D. Bartolomeo, L. Iemmo, and G. Luongo, Appl. Sci. 8, 526 (2018).

3 Z. Xiao, J. She, S. Deng, Z. Tang, Z. Li, J. Lu, and N. Xu, ACS Nano 4, 6332 (2010).

4 R. Diehl, M. Choueib, S. Choubak, R. Martel, S. Perisanu, A. Ayari, P. Vincent, S. T. Purcell, and P. Poncharal, Phys. Rev. B 102, 035416 (2020).

5 S. Santandrea, F. Giubileo, V. Grossi, S. Santucci, M. Passacantando, T. Schroeder, G. Lupina, and A. D. Bartolomeo, App. Phys. Lett. 98, 163109 (2011).

6 J. Xu, Q. Wang, Z. Tao, Z. Qi, Y. Zhai, S. Wu, X. Zhang, and W. Lei, ACS Appl. Mater. Interfaces 8, 3295 (2016).

7 J. Xu, Q. Wang, Z. Tao, Z. Qi, Y. Zhai, W. Lei, and X. Zhang, RSC Adv. 6, 78170 (2016).

8 A. Andronov, E. Budylina, P. Shkitun, P. Gabdullin, N. Gnuchev, O. Kvashenkina, and A. Arkhipova, J. Vac. Sci. Technol. B 36, $02 \mathrm{C} 108$ (2018).

9 R. H. Fowler and L. Nordheim, Proceedings of the Royal Society of London A: Mathematical, Physical and Engineering Sciences 119, 173 (1928).

10 L. W. Nordheim, Proc. R. Soc. London, Ser. A 121, 626 (1928).

11 R. Burgess, H. Kroemer, and J. Houston, Phys. Rev. 90, 515 (1953).

12 E. L. Murphy and R. H. Good, Physical Review 102, 1464 (1956).

13 J. He, P. H. Cutler, and N. M. Miskovsky, App. Phys. Lett. 59, 1644 (1991).

14 R. G. Forbes, App. Phys. Lett. , 89, 113122 (2006).

15 A. Modinos, Solid-State Electronics 45, 809 (2001).

16 R. G. Forbes, J. H. B. Deane, A. Fischer, and M. S. Mousa, Jordan Journal of Physics 8, 125 (2015).

17 X.-Z. Qin, W.-L. Wang, N.-S. Xu, Z.-B. Li, and R. G. Forbes, Proc. R. Soc. A 467, 1029 (2011).

18 Y. S. Ang, S.-J. Liang, and L. K. Ang, MRS Bulletin 42, 505 (2017).

19 Y. Ang and L. Ang, 31st International Vacuum Nanoelectronics Conference (IVNC), Kyoto, p. 1 (2018).

20 J. Peng, Z. Li, C. He, G. Chen, W. Wang, S. Deng, N. Xu, X. Zheng, G. Chen, C. J. Edgcombe, 
et al., Journal of Applied Physics 104, 014310 (2008).

21 M. Khazaei, A. A. Farajian, and Y. Kawazoe, Phys. Rev. Lett. 95, 177602 (2005).

22 Y. Gohda, Y. Nakamura, K. Watanabe, and S. Watanabe, Phys. Rev. Lett. 85, 1750 (2000).

23 Y. Gohda and S. Watanabe, Phys. Rev. Lett. 87, 177601 (2001).

24 Y. Gohda and S. Watanabe, Surface Science 516, 265 (2002).

25 Y. Gohda, Y. Nakamura, K. Watanabe, and S. Watanabe, Materials Science and Engineering A 327, 1 (2002).

26 Y. Gohda and S. Watanabe, J. Phys.: Condens. Matter 16, 4685 (2004).

27 S. F. Huang, T. Leung, B. Li, and C. Chan, Phys. Rev. B 72, 035449 (2005).

28 A. Mayer, N. M. Miskovsky, P. H. Cutler, and P. Lambin, Phys. Rev. B 68, 235401 (2003).

29 Z. Li, N. Xu, and H. J. Kreuzer, Phys. Rev. B 85, 115427 (2012).

30 P. Yaghoobi, K. Walus, and A. Nojeh, Phys. Rev. B 80, 115422 (2009).

31 S. Han, M. H. Lee, and J. Ihm, Phys. Rev. B 65, 085405 (2002).

32 Y.-W. Son, S. Oh, J. Ihm, and S. Han, Nanotechnology 16, 125 (2005).

33 C. Choong-Ki Lee, B. Lee, and S. Ihm, J. Han, Nanotechnology 18, 475706 (2007).

34 Y. Li, Y. Sun, and J. Yeow, Nanotechnology 26, 242001 (2015).

35 Z. B. Li, Ultramicroscopy 159, 162 (2015).

36 B. Lepetit, D. Lemoine, and M. Márquez-Mijares, J. App. Phys. 120, 085105 (2016).

37 B. Lepetit, J. Appl. Phys. 122, 215105 (2017).

38 B. Lepetit, J. App. Phys. 125, 025107 (2019).

39 J. Bardeen, Phys. Rev. Lett. 6, 57 (1961).

40 R. Ramprasad, L. R. C. Fonseca, and P. von Allmen, Phys. Rev. B 62, 5216 (2000).

41 N. Ngoc-Hieu, N. Van-Hanh, and T. Cong-Phong, Computational Materials Science 49, S218 (2010).

42 J. Bardeen, Phys. Rev. Lett., 6, 57 (1961).

43 S. C. Lam and R. J. Needs, J. Phys. : Condens. Matter 5, 2101 (1993).

44 N. D. Lang and W. Kohn, Phys. Rev. B 7, 3541 (1973).

45 A. G. Eguiluz, M. Heinrichsmeier, A. Fleszar, and W. Hanke, Phys. Rev. Lett. 68, 1359 (1992).

46 M. Abramowitz and I. A. Stegun, Handbook of mathematical functions (Dover, New York, 1965).

47 M. S. Child, Molecular collision theory (Academic Press, 1974).

48 A. H. Castro-Neto, F. Guinea, N. M. R. Peres, K. S. Novoselov, and A. K. Geim, Rev. Mod. 
Phys. 81, 109 (2009).

49 M. Katsnelson, Graphene. Carbon in two dimensions (Cambridge university press, 2012).

50 D. Niesner and T. Fauster, J. Phys.: Condens. Matter 26, 393001 (2014).

51 N. Armbrust, J. Güdde, and U. Höfer, New J. Phys. 17, 103043 (2015).

52 P. Puschnig, P. Amiri, and C. Draxl, Phys. Rev. B 86, 085107 (2012).

53 J. B. Neaton, M. S. Hybertsen, and S. G. Louie, Phys. Rev. Lett. 97, 216405 (2006).

54 C. Cohen-Tannoudji, B. Diu, and F. Laloë, Quantum mechanics (Wiley, 2005).

55 E. Clementi and D. L. Raimondi, J. Chem. Phys. 38, 2686 (1963).

56 Y. S. Ang, Y. Chen, C. Tan, and L. K. Ang, Phys. Rev. App., 12, 014057 (2019).

57 Y. S. Ang, C. H. Lee, and L. K. Ang (2021), URL https://arxiv.org/abs/2003.14004.

58 Y. S. Ang, L. Cao, and L. K. Ang, InfoMat., p. 1 (2021). 

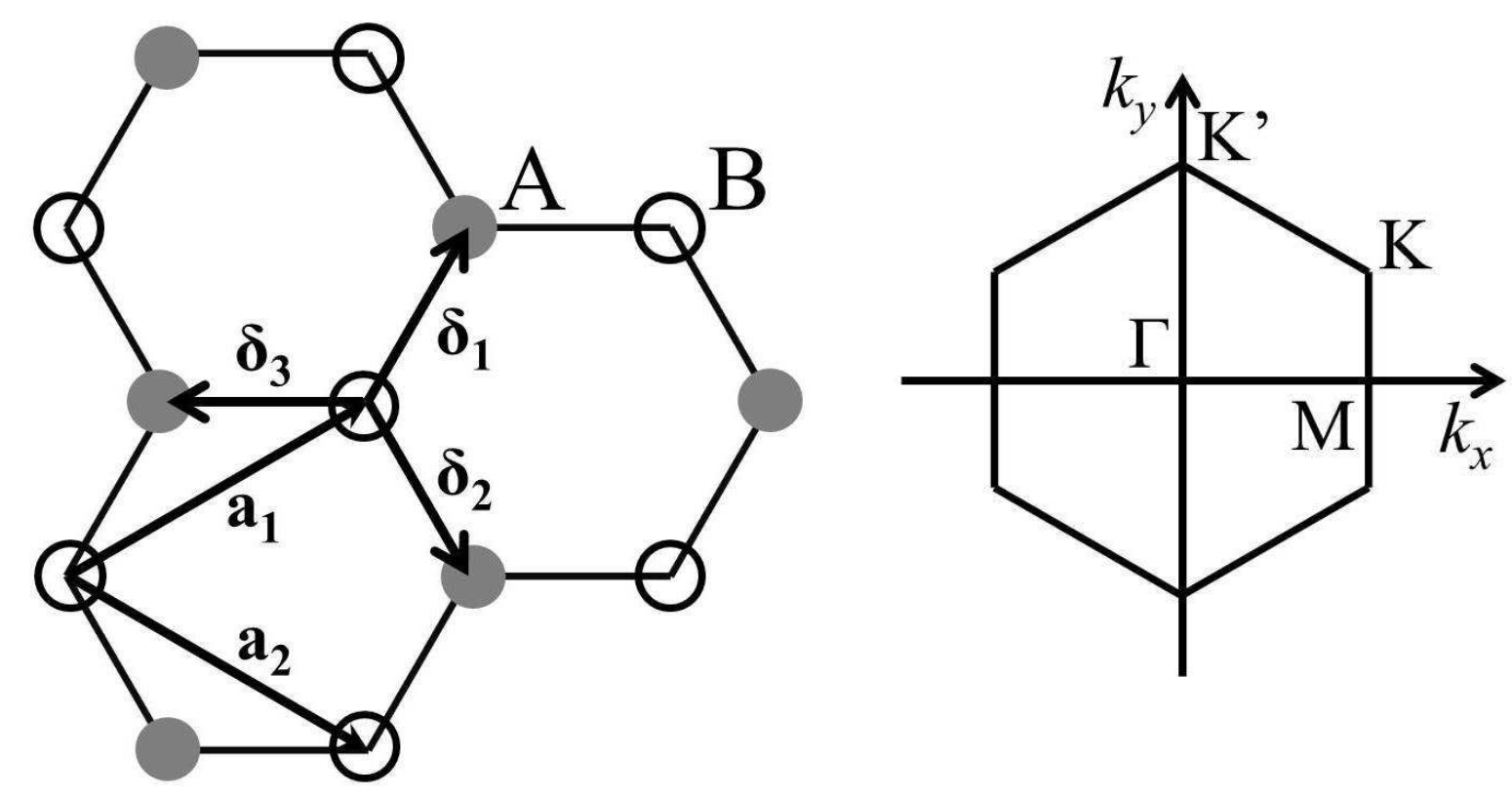

FIG. 1: Left : graphene lattice. Its primitive basis consists of 2 carbon atoms labelled A and B. A and B type atoms are connected separately by the lattice translational vectors $\mathbf{a}_{\mathbf{1}}$ and $\mathbf{a}_{\mathbf{2}} . \delta_{\mathbf{1}}, \delta_{\mathbf{2}}$ and $\delta_{\mathbf{3}}$ are vectors connecting nearest-neighbor atoms. Right : graphene first Brillouin zone (1BZ) and selected points in reciprocical space : $\Gamma\left(\mathbf{k}_{\Gamma}=(0,0)\right), \mathrm{M}\left(\mathbf{k}_{M}=\left(\frac{2 \pi}{3 a}, 0\right)\right), \mathrm{K}\left(\mathbf{k}_{K}=\left(\frac{2 \pi}{3 a}, \frac{2 \pi}{3 \sqrt{3} a}\right)\right)$ and $\mathrm{K}^{\prime}\left(\mathbf{k}_{K^{\prime}}=\left(0, \frac{4 \pi}{3 \sqrt{3} a}\right)\right)$. K and $\mathrm{K}^{\prime}$ are Dirac points. 

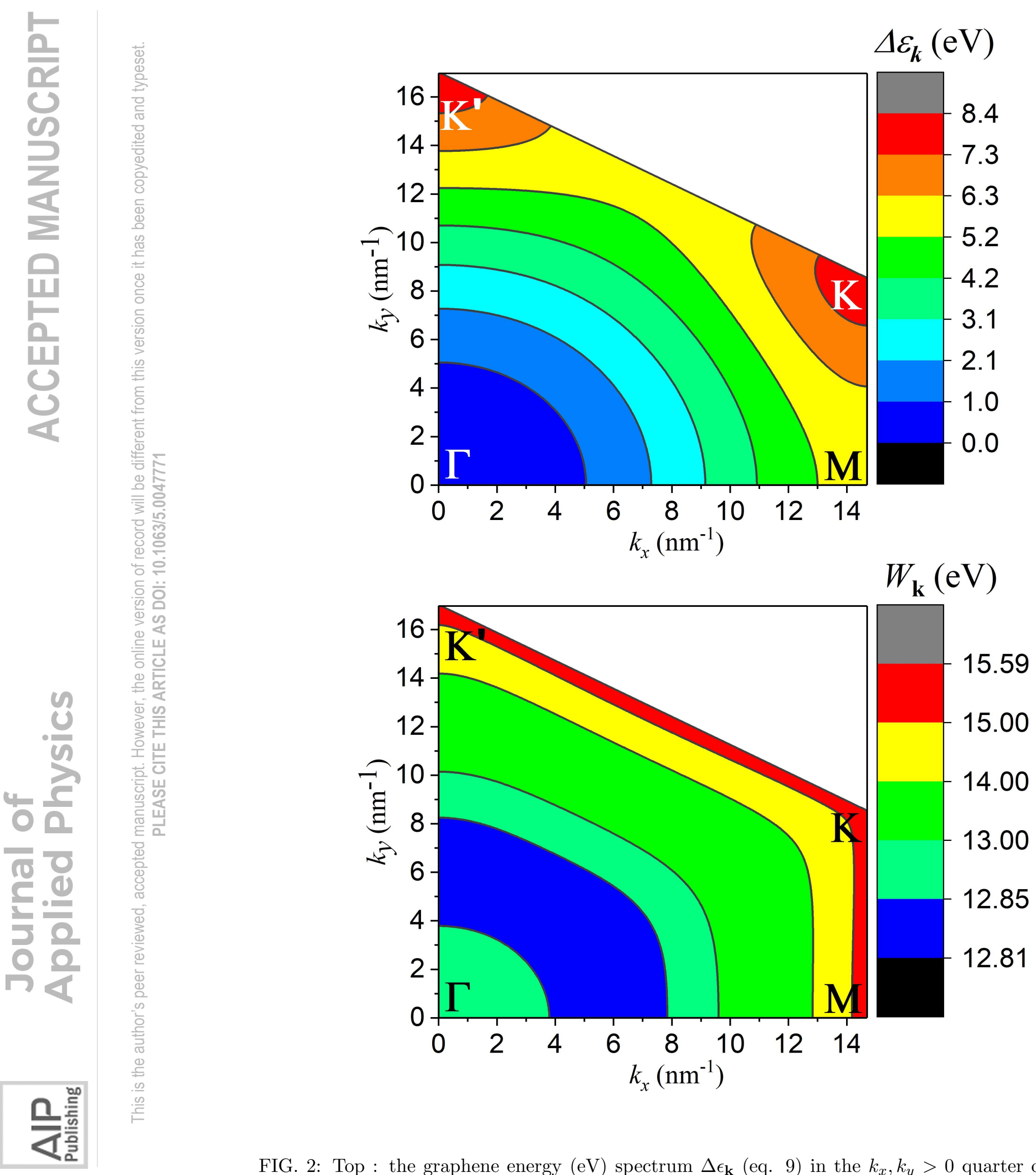

FIG. 2: Top : the graphene energy (eV) spectrum $\Delta \epsilon_{\mathbf{k}}$ (eq. 9) in the $k_{x}, k_{y}>0$ quarter of the 1BZ. Bottom : The energy (eV) $W_{\mathbf{k}}$ (eq. 9) necessary to extract an electron with momentum $\mathbf{k}_{\mathbf{x y}}$ from the graphene energy level $\epsilon_{\mathbf{k}}$ into the vacuum energy level $V_{\infty}+\frac{\hbar^{2} \mathbf{k}_{\mathbf{x y}}}{2 m}$. 


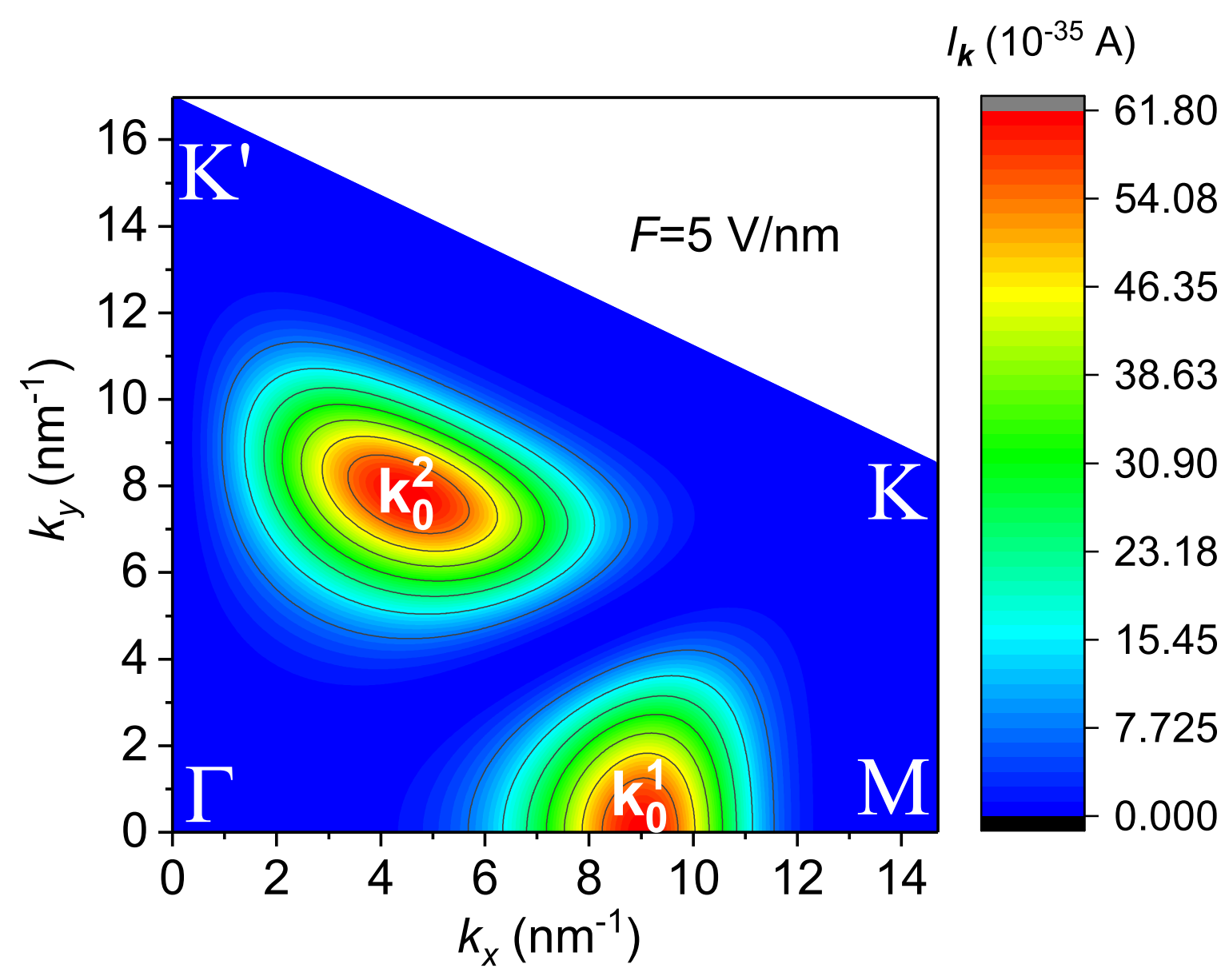

FIG. 3: Emitted current distribution $I_{k}$ (eq. 20) in the $k_{x}, k_{y}>0$ quarter of the $1 \mathrm{BZ}$ for $F=5$ $\mathrm{V} / \mathrm{nm}$. Two 1BZ maximum emission points $\mathbf{k}_{0}^{\mathbf{i}}(\mathbf{i}=1-2)$ are also shown. 


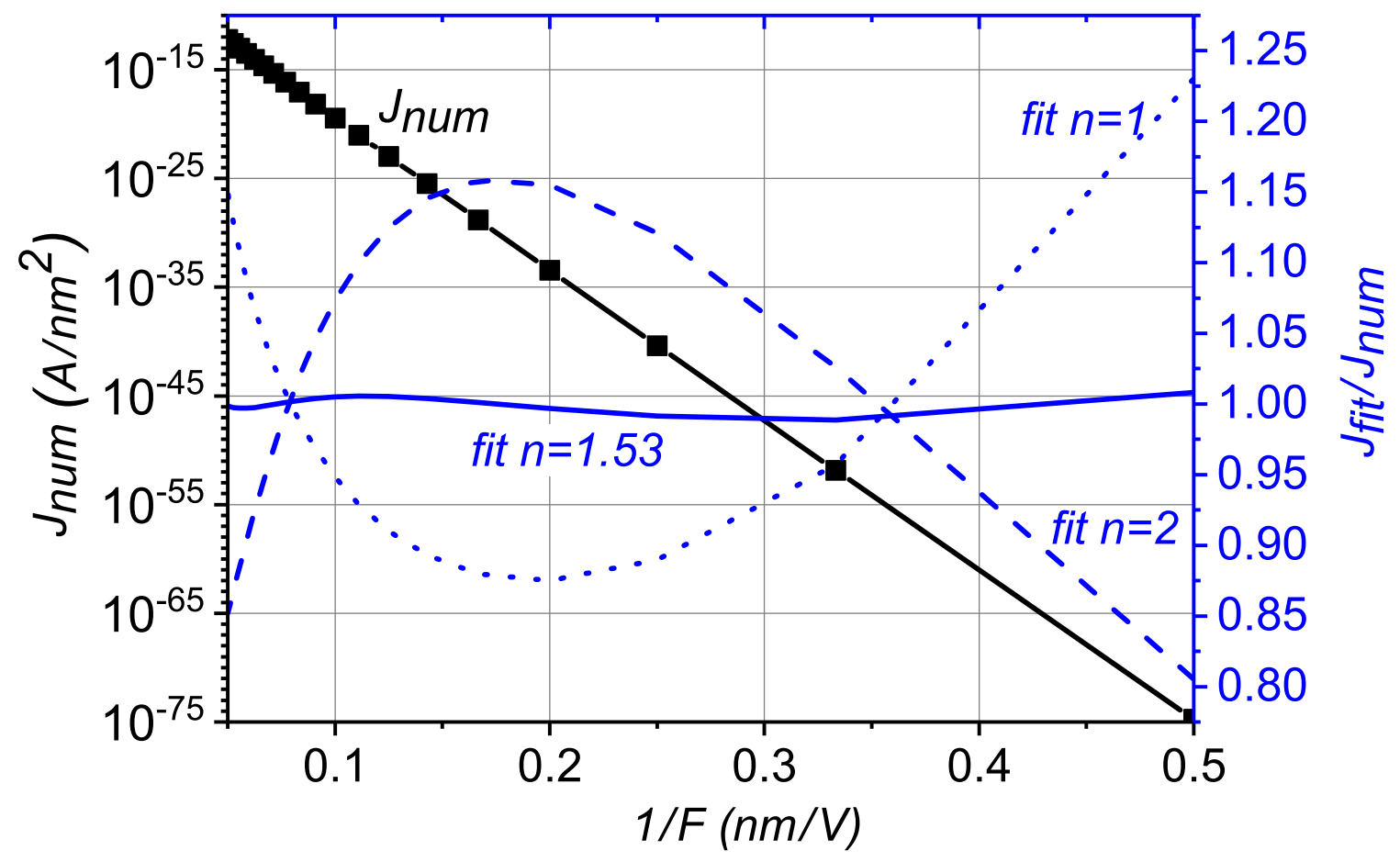

FIG. 4: Full black line connecting symbols (left scale, $A / n m^{2}$ ) : emitted current density $J_{\text {num }}$ as the function of the applied electric field $F(V / n m)$, obtained by numerical integration (eq. 3 and 20). Blue lines (right scale) : ratios between fitted and numerical results $J_{f i t} / J_{n u m}, J_{f i t}$ being given by eq. 14. Dotted line : fixed parameter $n=1$, fit parameter $W=12.86 \mathrm{eV}$. Dashed line : fixed parameter $n=2$, fit parameter $W=12.71 \mathrm{eV}$. Full line : fit parameters $n=1.53$ and $W=12.78 \mathrm{eV}$. 


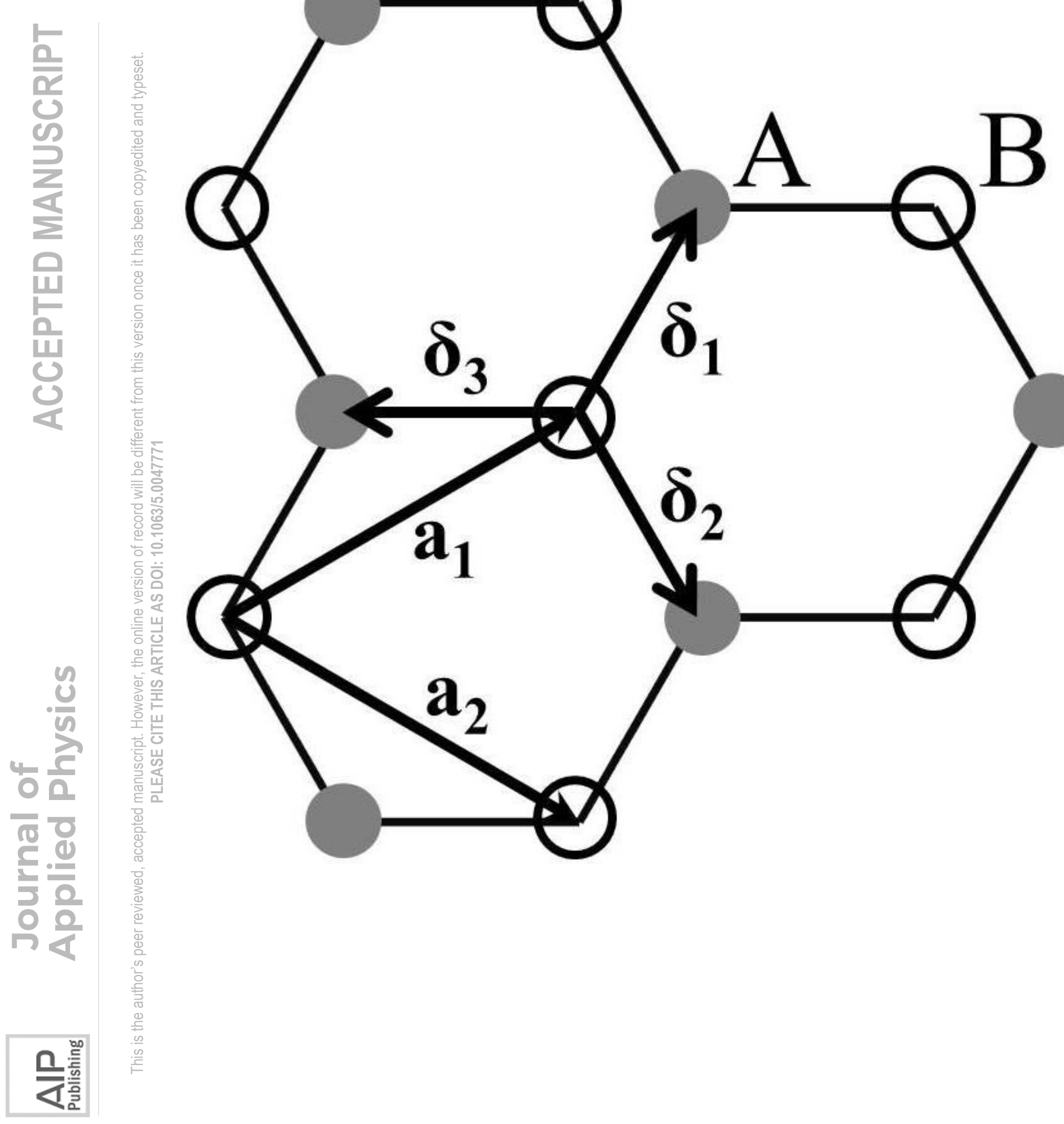




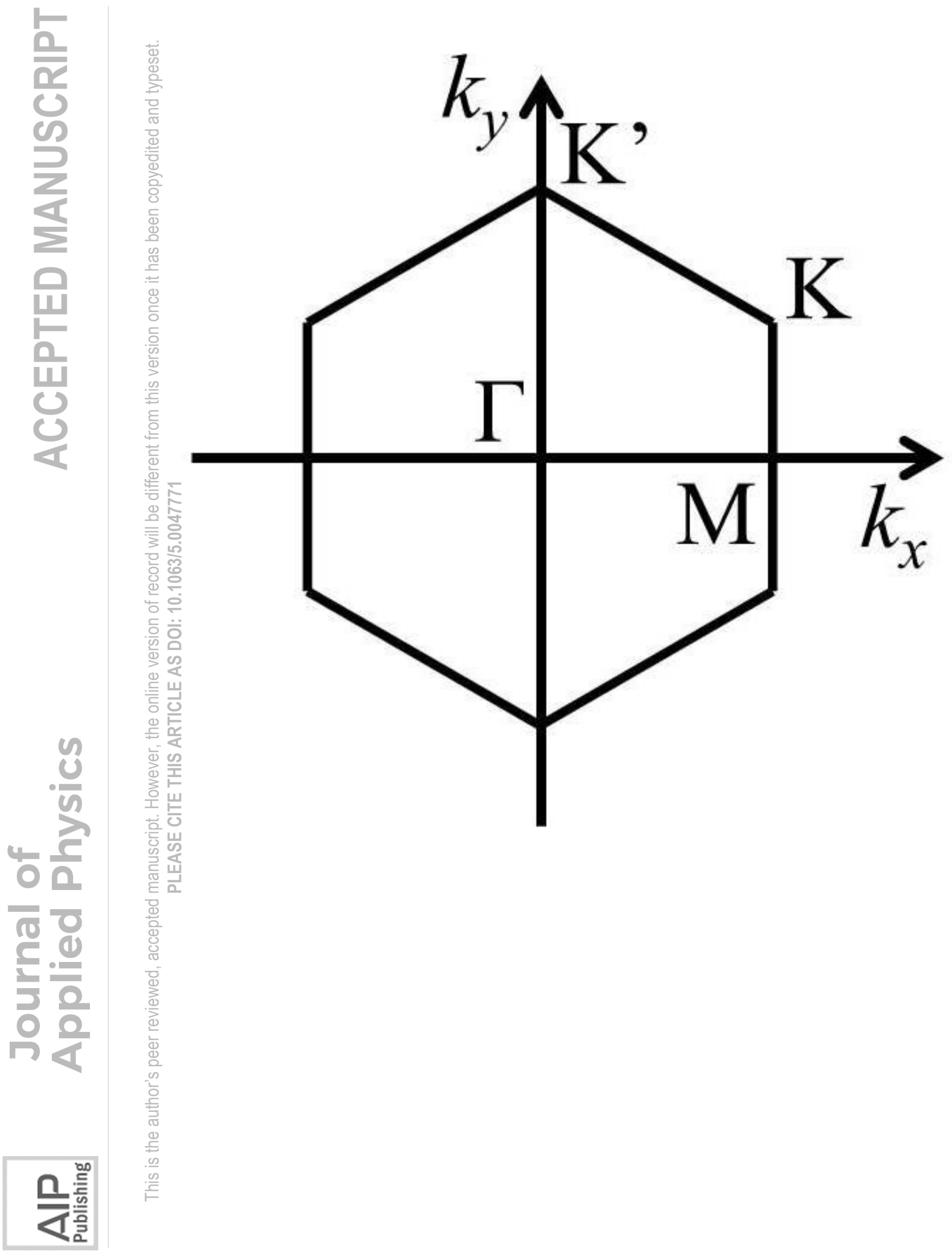


$W_{\mathbf{k}}(\mathrm{eV})$

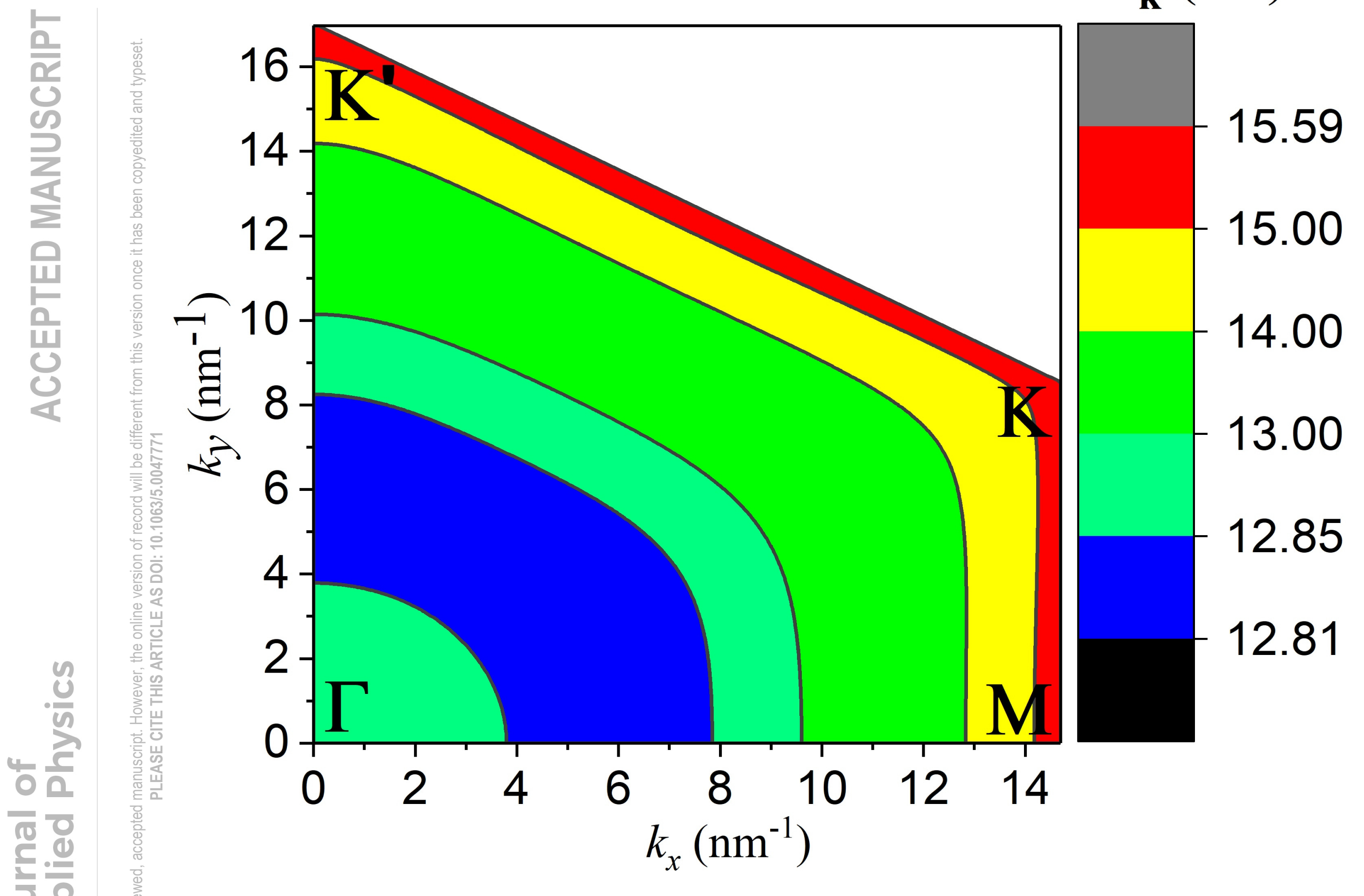


$\Delta \varepsilon_{\boldsymbol{k}}(\mathrm{eV})$

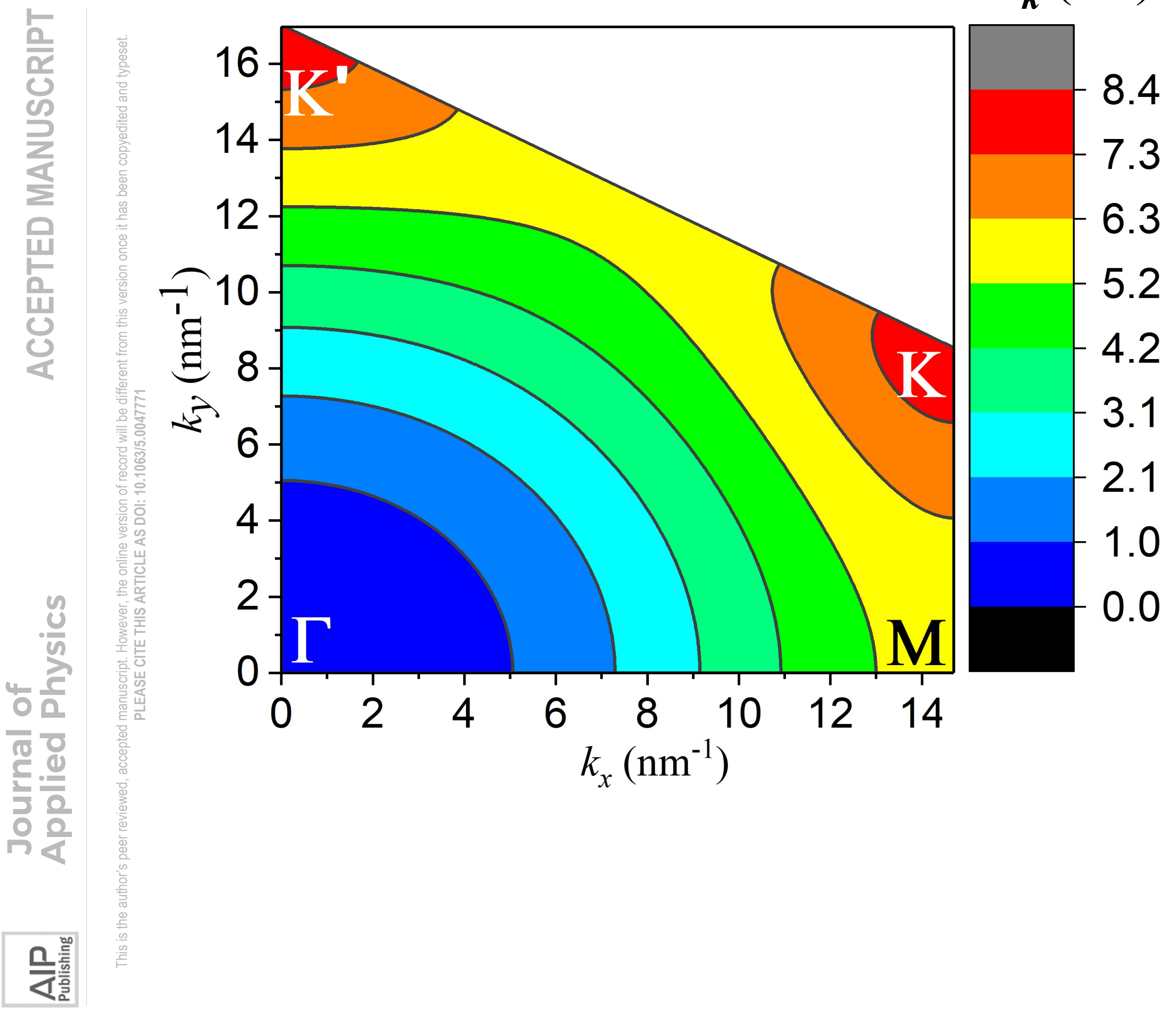




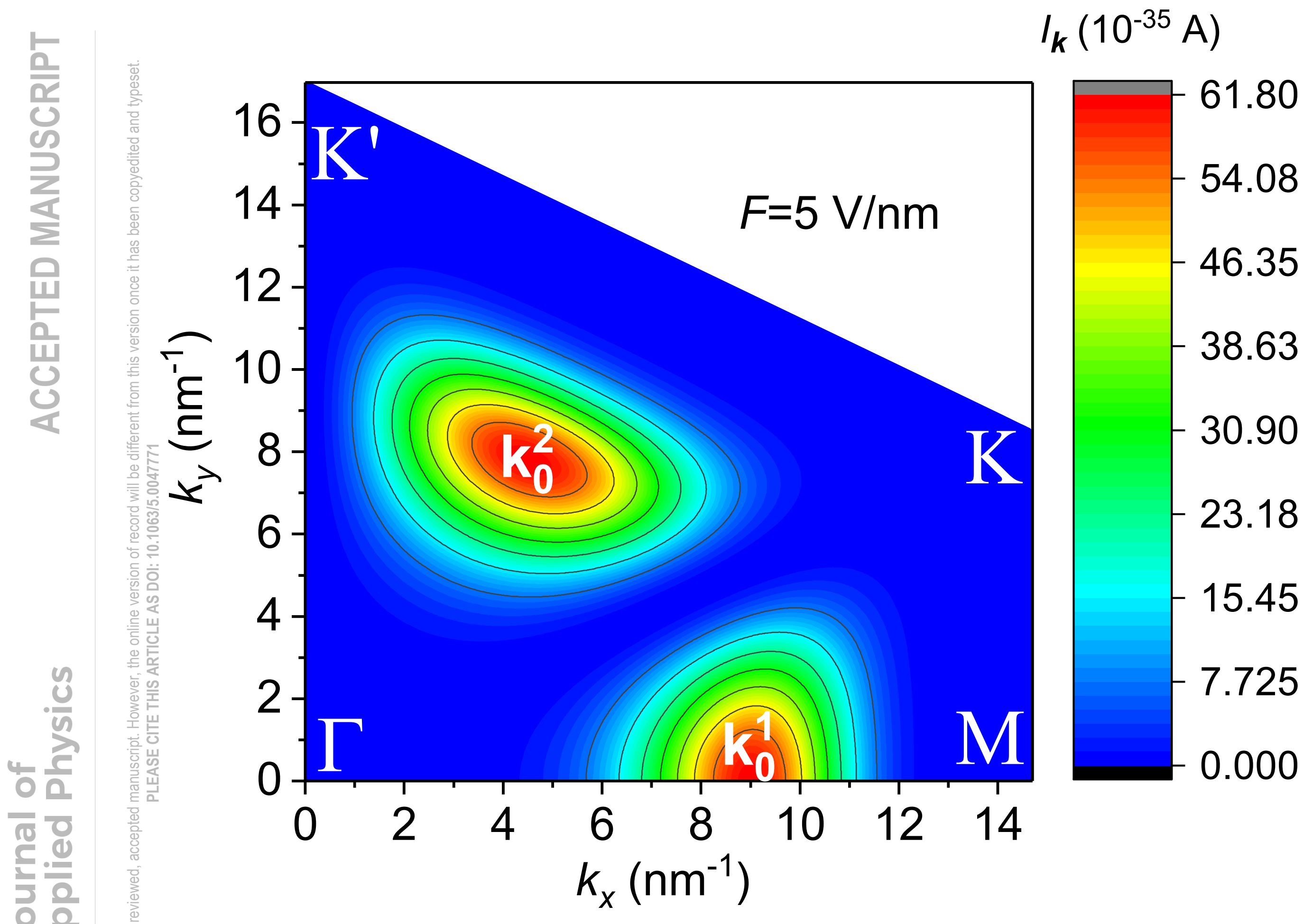




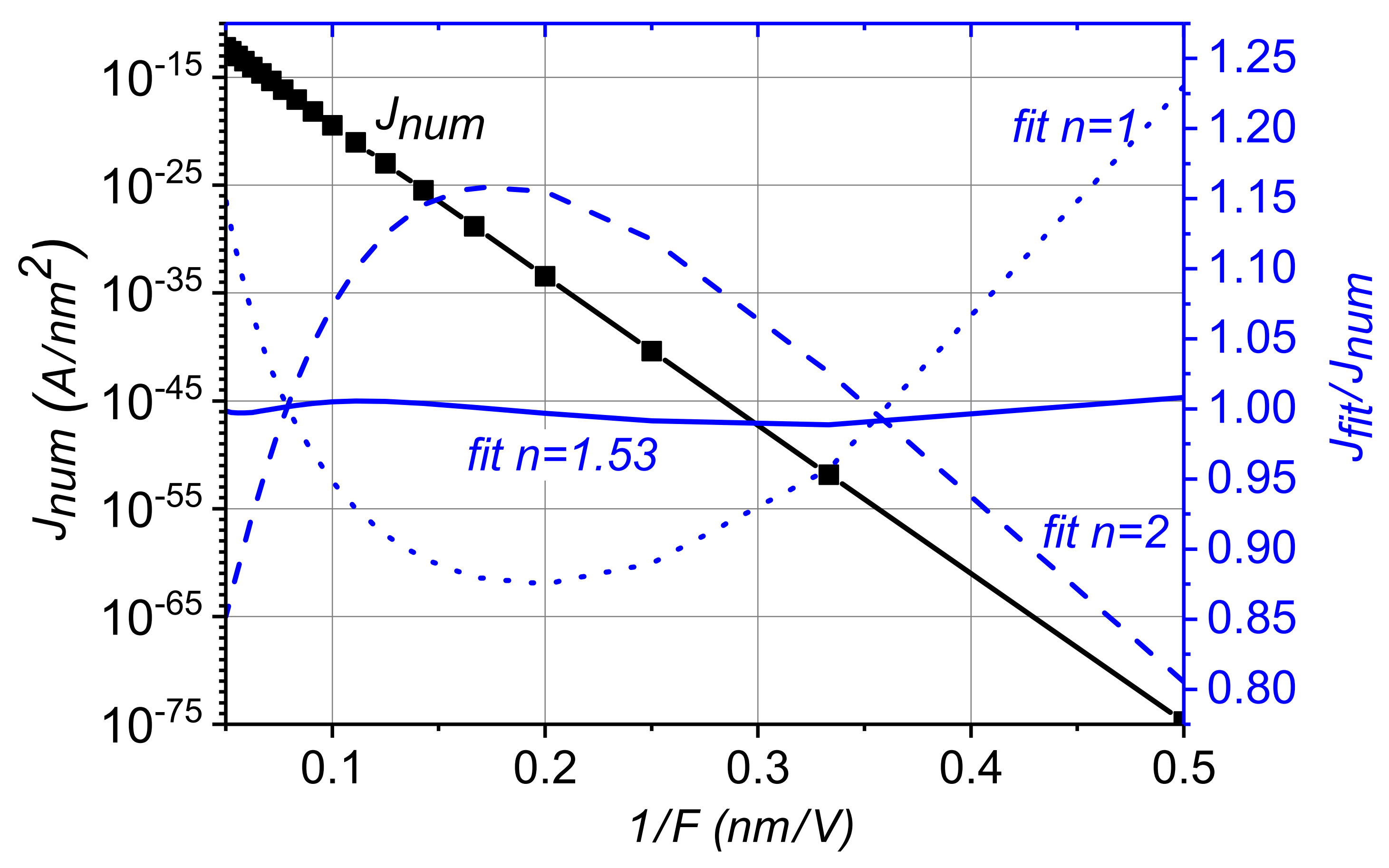

